\title{
EDUCATION AND TRAINING Haematology morphology teaching during the COVID-19 pandemic: a UK teaching hospital experience
}

\author{
Authors: Sarah K Westbury ${ }^{A}$ and Andrew Stewart ${ }^{B}$
}

\begin{abstract}
Postgraduate medical education faces ongoing disruption due to the COVID-19 pandemic, with specific challenges in providing training in laboratory medicine. We report an online approach that has enabled us to continue to deliver haematology morphology training in a teaching hospital setting. The method described is associated with high levels of participant satisfaction and could easily be adapted for use in other clinical pathology specialties.
\end{abstract}

KEYWORDS: haematology, COVID-19, education, microscopy

DOI: $10.7861 /$ fhj.2020-0159

\section{Background}

Training in blood and bone marrow cell morphology is an essential component of postgraduate haematology medical education. It is perceived as a challenging area in which to both obtain and maintain skills. ${ }^{1}$ Gaps in training rotas, the European Working Time Directive, subspecialty training and other logistical or staffing issues may all impact on the quantity and quality of morphology training available for haematology trainees. ${ }^{2}$ Here we describe our experiences of delivering haematology morphology teaching during the COVID-19 pandemic in a large UK NHS trust.

\section{Problem}

Postgraduate medical education has been significantly disrupted by the COVID-19 pandemic, both due to alterations in trainee working patterns and the need for social distancing in the workplace. Other healthcare systems that have previously been affected by disease epidemics have arguably been better prepared to deal with the impact on postgraduate training and have moved rapidly to incorporate online learning. 3,4

Prior to the COVID-19 pandemic, morphology teaching at University Hospitals Bristol and Weston NHS Foundation Trust (UHBW) consisted of a weekly, hour-long teaching session

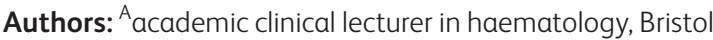
Royal Infirmary, Bristol, UK and University of Bristol, Bristol, UK; ${ }^{B}$ consultant haematologist, Bristol Royal Infirmary, Bristol, UK regularly attended by training and non-training middle-grade haematology doctors. A small number of biomedical scientists and consultants with laboratory responsibilities also attended intermittently. Blood films, bone marrow aspirates and trephines were reviewed around a multiheaded (five-head) training microscope (EXC-500; Accu-Scope, Commack, USA) with a digital camera and large high-resolution screen to enable other participants in the laboratory to view the material simultaneously. This arrangement did not allow for adequate social distancing within the laboratory, and so morphology teaching was not possible even when clinical pressures had eased sufficiently to allow postgraduate education events to recommence.

\section{Solution}

Our solution to this was to redesign morphology training within UHBW using a remote, online teaching approach, with synchronous and asynchronous components. Synchronous teaching refers to teaching that participants experience at the same time but in a different place, while asynchronous teaching occurs at a different time and a different place for participants. ${ }^{5}$ This can also be combined with more traditional face-to-face training opportunities to produce a blended learning experience. ${ }^{6}$

Five days prior to the morphology teaching session, anonymised clinical material relating to one or two cases is circulated to participants via e-mail. This typically includes a clinical vignette and basic full blood count information in addition to between two and eight digital images of blood or marrow slides for examination. Images at different magnifications are selected that demonstrate the key morphological features of the case. Depending on the scenario, additional information such as flow cytometry plots, high performance liquid chromatography traces and cytogenetic or genomic results may also be included. Participants are asked to review this material (asynchronous component) prior to the weekly morphology teaching (synchronous component). The weekly synchronous teaching is delivered via Webex videoconferencing (Cisco, London, UK). Webex is used as it has full IT support from UHBW and is the same videoconferencing system as used for the virtual haematology pathology multidisciplinary team meetings (MDTs). The person leading the teaching session uses the screen share function to enable all participants to view the material, and participant interaction is possible either verbally or using the chat function 
for those without microphones. The teaching starts with a live review of the slides and other material sent out before considering previously unseen blood film and bone marrow slides, with participants taking turns to describe the morphological features, to suggest a diagnosis or differential diagnosis, and to identify what further tests would be indicated. During the synchronous teaching, participants see the same 'view' on their screen as the facilitator sees through the microscope. Participants are therefore able to direct the facilitator to navigate around the slide, focus on a particular cell or feature, or change objective lens. The images viewed on a computer or tablet in real time are clear and of comparable quality to what can be seen using the microscope, but participants joining via their mobile phone may have a less satisfactory experience due to screen size.

\section{Results so far}

The new teaching method has been well received by permanent staff and trainees at UHBW and resulted in two nominations for a UHBW Spotlight Award, recognising the beneficial impact of this virtual training. Feedback via free-text responses to semistructured questions circulated by e-mail and in paper format has been universally positive from both training and non-training grade haematologists. Participation rates were higher for the remote teaching than they were for the face-to-face morphology teaching, with more biomedical scientists and clinical scientists attending, as well as higher attendance rates among the middle grade doctors. As participants can join from any location, we have particularly noticed an increase in attendance by those who work across multiple sites in the trust, those who work flexibly and those who work less than full time. Furthermore, individuals who are not in work due to COVID-19-specific issues (such as shielding and self-isolation) have still been able to participate in the teaching. Attendance numbers increased from an average of approximately seven participants for the pre-pandemic, in-person teaching to approximately 20 participants for the virtual teaching. Despite the increase in participant numbers, the sessions remain highly interactive. For the facilitators, the interactive nature of the teaching avoids the sensation of 'teaching to the void' that can accompany the delivery of online lectures or other larger group formats.

The main negative element identified so far with this approach is the increase in time required to prepare and deliver the teaching. Preparation of digital material for circulation in advance of each teaching session takes additional time but can be combined in some instances with preparation for MDTs or laboratory quality assurance exercises. Although there have been relatively few practical issues so far, the synchronous sessions work most smoothly when the facilitator leading the session has an assistant to help with monitoring the chat function, responding to queries or dealing with any technical problems. The key advantages and disadvantages of the virtual method described here are outlined in Table 1.

\section{Further evaluation}

As a result of participant feedback, the teaching session has been opened to all haematology trainees in the region in addition to UHBW trainees and staff. The remote morphology teaching described here has been delivered predominantly to haematology

Table 1. The major advantages and disadvantages

of the online approach to morphology teaching

\section{Advantages of online approach}

Ability to continue with morphology training

Enables participation of those unable to attend in-person training (eg those shielding or in selfisolation)

Increased numbers participating

More interdisciplinary training and discussion

Use of the asynchronous material to prepare may encourage participation of less confident trainees

Similar experience to participation in virtual MDTs and other clinical meetings

MDTs $=$ multidisciplinary team meetings.

trainees and middle grades with some experience of microscope work and pre-existing morphology knowledge. The months of August and September see a number of new specialty trainees join the haematology training programme, and it will be important to ensure that the training is accessible and fit for purpose for those trainees with limited or no previous morphology experience. For these individuals, a blended approach to learning will be crucial to ensure they develop the necessary microscopy skills alongside recognition of any pathological findings. Clinical and educational supervisors will have key roles in ensuring that this approach meets the needs of junior trainees, as indeed they will for all aspects of haematology training that have been influenced by the pandemic. In addition, the remote nature of the teaching has led to the participation of a small number of non-physician, non-laboratory healthcare professionals, principally haematology specialist prescribing pharmacists. Further evaluation of the teaching will be required to ensure that we are obtaining the benefits of multiprofessional training while not compromising the core aim of ensuring that clinical haematologists receive adequate training and continuing professional development in this core diagnostic skill. Future feedback questionnaires will need to be carefully formatted to ensure that the experiences of the small numbers of early trainees and non-haematologists are adequately captured without inadvertently identifying them, and that the needs of trainees based in other NHS trusts are being adequately met.

\section{Potential future}

Even as restrictions secondary to COVID-19 lift, we anticipate maintaining the morphology teaching in a remote, online form. 
Facilitators and participants alike are becoming more familiar with the virtual learning environment, and changes in the delivery of training due to COVID-19. Including zoomable or browsable digital images, such as those used by the NEQAS digital morphology scheme (www.ukneqash.org/dm), would be a valuable future development and one that we hope to incorporate in the near future. It will also be important to ensure that any changes in the format of the haematology FRCPath Part 2 examination (such as the inclusion of digital morphology examinations) are reflected in the teaching. As trainees rotate through laboratory haematology at UHBW and other trusts in the region, the overall morphology learning experience will be blended, with microscopy training in their host trusts as well as centrally delivered online morphology teaching. We believe this blended approach could be readily adapted for use in other clinical pathology specialties.

\section{References}

1 Hamilton PW, van Diest PJ, Williams R, Gallagher AG. Do we see what we think we see? The complexities of morphological assessment. J Pathol 2009;218:285-91.
2 Shlebak AA, Bain BJ. Training future haematologists, a privilege or a burden? "A trainer's view". Br J Haematol 2017;178:501-7.

3 Cheong MA, Chee YL, $\mathrm{Ng} \mathrm{H}$ ], Jen WY. Keeping the bloodline of haematology medical education flowing during a pandemic. Int $]$ Lab Hematol 2020;42:e196-8.

4 Liang ZC, Ooi SBS, Wang W. Pandemics and their impact on medical training: lessons from Singapore. Acad Med 2020;95:1359-61.

5 Stewart CM, Schifter CC, Selverian MEM. Teaching and learning with technology: Beyond constructivism, 4th edn. New York: Routledge, 2010.

6 Westerlaken M, Christiaans-Dingelhoff I, Filius RM et al. Blended learning for postgraduates; an interactive experience. BMC Med Educ 2019;19:289.

Address for correspondence: Dr Sarah Westbury, Research Floor Level 7, Bristol Royal Infirmary, Upper Maudlin Street, Bristol BS2 8HW, UK.

Email: sarah.westbury@bristol.ac.uk 